\title{
¿CÓMO CREAN LOS SERES HUMANOS? \\ UNA APROXIMACIÓN NEUROPSICOLÓGICA Y NEUROBIOLÓGICA \\ A LA CREATIVIDAD ${ }^{1}$
}

\section{How do Human Beings Create? A Neuropsychological and Neurobiological Approach to Creativity}

\author{
Jaime Carrizosa Moog ${ }^{2}$
}

Recibido: 2018-10-22 Aceptado: 2018-12-20

\begin{abstract}
Resumen: El presente estudio pretende revisar las bases neuropsicológicas y neurobiológicas que subyacen al proceso de creatividad en el ser humano, considerando los resultados de estudios recientes sobre neuroanatomía funcional, genética y valoraciones neuropsicológicas. Mediante la reflexión crítica de estos resultados se pretende motivar al lector a la actualización de estos conocimientos, la aplicación de los mismos y la posible formulación de nuevas preguntas de investigación. De ese modo, la creatividad se puede medir con pruebas que incentivan el pensamiento divergente o el discernimiento interior. Los estudios de neuroimágenes funcionales aclaran con precisión las funciones cognitivas necesarias para la creatividad al localizarlas en estructuras cerebrales y demostrando su interrelación. Así
\end{abstract}

pues, son útiles en el proceso de creatividad: la motivación, la apertura a lo novedoso, la inteligencia, la inspiración y la imaginación. Existen genes identificados que están relacionados con la creatividad. Igualmente, la creatividad se puede incentivar con la marcha, salidas al campo, ocio, reuniones multiculturales y el sueño. Los resultados expuestos en el presente estudio requieren replicación y análisis en contextos diferentes; así, se pueden abrir novedosas oportunidades de aplicación en muchas disciplinas y una apertura a nuevas preguntas de investigación sobre creatividad que permitan aclarar las bases funcionales y eventual traslación a la práctica cotidiana.

Palabras clave: creatividad, pensamiento, intuición, neuroimagen, inteligencia.
Para citar este artículo en APA: Carrizosa, J. (2018). ¿Cómo crean los seres humanos? Una aproximación neuropsicológica y neurobiológica a la creatividad. Revista de Psicologia Universidad de Antioquia, 10(2), 183-202. DOI: 10.17533/ udea.rp.v10n2a08
Revisión de tema.

2. Especialista en Neurología Infantil. Profesor titular, Facultad de Medicina, Departamento de Pediatría y Puericultura, Universidad de Antioquia, Medellín, Colombia. Correo: jaime. carrizosa@udea.edu.co; http://orcid.org/0000-0002-36517285 
Abstract: Creativity is a new and useful cognitive product in a given social context. Recent genetic, functional neuroimaging and neuropsychological studies achieved interesting results regarding the anatomic and functional mechanisms that underlay creativity. A critical appraisal should serve as an update and enforcement of knowledge so as the possible amplification of research questions. Creativity can be measured with tests inspiring divergent thinking or insight. Functional neuroimaging and new genetic testing and interpretation are giving clues in cerebral anatomic localization and interrelation of cognitive tasks during creativity. Factors like inte- lligence, motivation, imagery, inspiration and openness to new experiences boost creativity. Multicultural experiences, walking, enjoying green leisure time, looseness and dreaming can elucidate creative thinking. Although the new data require replication in other contexts, novel application opportunities in different disciplines appear. Also intriguing research questions in the field could clarify the underlying functions of creativity and its translation to daily practice.

Keywords: creativity, thinking, insight, neuroimaging, intelligence.

\section{Introducción}

La creatividad se define como el trabajo que produce algo novedoso, inesperado y original, útil en un contexto social determinado (Dietrich, 2004; Flaherty, 2005; Jung et al., 2010). Dicho trabajo compromete tanto a las ciencias como a las artes y es un área de confluencia de muchas disciplinas. La ciencia cognitiva estudia la creatividad como una función cerebral y la explica desde dimensiones funcionales y anatómicas como la intencionalidad emocional, inteligencia, metacognición, memoria-aprendizaje, flexibilidad cognitiva, evaluación, introspección, entre muchos otras (Carlsson, Wendt, y Risberg, 2000; Mula, Hermann y Trimble, 2016). Se pretende revisar las bases neuropsicológicas y neurobiológicas que subyacen al proceso de creatividad en el ser humano considerando los resultados de estudios recientes sobre neuroanatomía funcional, genética y valoraciones neuropsicológicas. Mediante la reflexión crítica de estos resultados se pretende motivar a artistas, científicos y profesionales que trabajan con procesos creativos, en la actualización de estos conocimientos, la aplicación de los mismos y la posible formulación de nuevas preguntas de investigación. 


\section{Desarrollo}

\section{Mediciones de la creatividad}

El psicólogo Joy Paul Guilford estableció en la década de 1940 el concepto de pensamiento divergente como una función mental medible de la creatividad (Lehmann, 2018). Empero, en contraposición a esta forma de razonar se encuentra el pensamiento analítico, deductivo, que aplica la convergencia de ideas y acciones para solucionar algún problema cotidiano. Mediante diversas pruebas se plantea una situación u objeto, a la cual se le debe dar múltiples salidas o funciones posibles y viables que no necesariamente son las usuales o socialmente correctas. Se puede, por ejemplo, incentivar las innumerables funciones que puede tener un zapato o las terminaciones diversas de un cuadro partiendo sólo de unas pocas líneas plasmadas en un papel. Ejemplos de estas pruebas son el test de uso inusual —Unusual Uses Test- o el test de Torrance de pensamiento creativo — Torrance Test of Creative Thinking-, que tiene categorías de exploración verbal y visual. Otras pruebas incitan el discernimiento de la persona para resolver asociaciones o adivinanzas como métodos para medir la creatividad: es el caso de la tarea de asociación remota —Remote Association Task —. También existen pruebas que miden la creatividad en diferentes áreas como literatura, escultura, pintura e inclusive matemáticas. Otra prueba, como el test de logros creativos —Creative Achievement Test_, alienta la producción de ideas en diversos terrenos del conocimiento, determinando el potencial creativo de un individuo en general y no sólo dentro un área específica.

Es interesante saber que existen pruebas que miden la creatividad, fenómeno que pudiera considerarse individual, personal, casi íntimo; por lo que la pena preguntarse si las pruebas descritas consideran variables que influyen en el desarrollo, calidad y valoración de los productos finales. Algunas de estas variables podrían estar relacionadas con la originalidad del producto final y con la personalidad, inteligencia y emocionalidad del evaluado. También se puede cuestionar si una acción subjetiva, como lo es de cierta forma la creatividad, 
puede someterse a pruebas de valoración estrechas con parámetros preestablecidos o con respuestas dirigidas o únicas.

En un mundo contemporáneo con un desarrollo tecnológico inusitado y veloz es cuestionable la validación de pruebas que no incluyen necesariamente las nuevas herramientas digitales, analógicas, convergentes y de simulación utilizadas con frecuencia en las ciencias y las artes. Quizás sea necesario construir nuevas pruebas acorde con el contexto de evolución de las artes, las ciencias y las disciplinas relacionadas con actividades creativas. La división de las pruebas en verbales y no verbales puede ser un ejemplo de la limitación de las mismas; para todos es conocido cómo en la apreciación de obras artísticas pueden confluir diversos sentidos y sensaciones. A manera de ejemplo, cabe la situación sobre si se ha apreciado determinada canción y se recibe la respuesta de que no se ha mirado el video.

\section{Creatividad e inteligencia}

Los investigadores difieren con respecto a la integración entre inteligencia y creatividad: unos consideran que son fenómenos estrechamente interconectados, mientras que otros argumentan que se tratan de fenómenos independientes. Los ensayos clínicos con neuroimagen funcional dan luces sobre este dilema:

- El estudio de Jung y sus colaboradores (2009) dirime este asunto mediante la medición de actividad neuronal de $\mathrm{N}$-acetil aspartato — NAA— en personas con inteligencia normal y superior. Se demuestra que en personas creativas e inteligencia normal - IQ $<116$ - disminuye la expresión de NAA en la sustancia gris frontal derecha. Las personas más inteligentes y creativas - IQ > 116 - se diferenciaban por un incremento de NAA en la corteza frontal izquierda. Estas observaciones dan indicios de que la inteligencia y la creatividad son fenómenos interrelacionados en las personas con inteligencia normal, pero que podrían ser procesos neurocognitivos independientes en personas con inteligencia superior pues activan áreas cerebrales diferentes.

- El flujo sanguíneo cerebral en reposo se incrementa significativamente en la región perisilviana izquierda en personas inteligentes, en tanto se dis- 
minuye en el precuneo en personas creativas, lo cual corrobora localizaciones anatómicas y funcionales diferentes entre creatividad e inteligencia en el cerebro (Takeuchi et al., 2011).

- Al realizar pruebas de analogías verbales, que requieren habilidades intelectuales y creativas, cabe resaltar la activación bilateral de los giros frontales superior, inferior y medio y de la corteza del cíngulo en la resonancia nuclear funcional. Estas zonas se han asociado con actividades de razonamiento abstracto como silogismos, sintaxis y creatividad lingüística (Geake y Hansen, 2005).

Con las nuevas herramientas diagnósticas e investigativas se abre el espacio para explorar los diferentes conceptos de inteligencia y su posible relación con la creatividad. Sería menester observar la relación que pudiera tener el funcionamiento cerebral de personas con diversos tipos de inteligencia — como emocional, comunicativa o atlética - frente a pruebas respectivas o compararlas con los resultados de las personas con inteligencia medida exclusivamente de manera psicométrica. Otro aspecto no menos relevante pudiera ser el de explorar las áreas de activación cerebral en personas con discapacidad cognitiva y su respectivo proceso de creatividad, lo cual puede dar luces sobre mecanismos de estimulación de dichas áreas en beneficio de las personas afectadas.

\section{Apertura a nuevas experiencias}

El rasgo de personalidad de apertura a nuevas experiencias pareciera ser una condición incitadora de la creatividad. Los estudios de neuroimagen funcional localizan este rasgo en la corteza frontal dorsolateral. Dietrich propone que gran parte del proceso de información intelectual se ubicaría en la corteza de asociación temporo-occipito-parietal, mientras que las funciones creativas se mediarían en la corteza prefrontal dorsolateral. Incluso expone una interrelación entre las dos áreas: una área de asociación muy fuerte con una corteza prefrontal débil conduciría a un mayor conocimiento - datos- con poco alcance de la creatividad - menos ideas nuevas-; por el contrario, una corteza prefrontal desarrollada con un área de asociación normativa o limitada generaría muchas ideas o propósitos a partir de pocos conocimientos o da- 
tos. Se sustenta la localización topográfica de las funciones intelectuales en las áreas de asociación, al confirmarse que las pruebas de inteligencia no se alteran de manera significativa con las lesiones frontales (Dietrich, 2004; DeYoung, Peterson y Higgins, 2005; Miller y Tal, 2007).

Existen estudios que demuestran cómo la apertura a experimentar situaciones nuevas está relacionada con el sistema dopaminérgico en las funciones de pulsión/recompensa entre el núcleo accumbens y la corteza frontal dorsolateral, el sistema atencional y de función ejecutiva, y la probable reducción de la inhibición latente en el lóbulo frontal. La combinación de estas funciones podría facilitar el paso de estímulos e ideas, los cuales amplían la fuente de percepciones y conocimientos necesarios para la creatividad; una función diferente de estas estructuras filtra, selecciona y suprime determinadas percepciones y pensamientos, lo que podría limitar la creatividad.

Un estudio con neuroimágenes funcionales en niños de China asoció un comportamiento impulsivo de toma de riesgos y desafíos durante la actividad creativa con cambios volumétricos de la sustancia gris de la amígdala y del hipocampo. También observaron que el incremento del volumen regional de la corteza orbitofrontal se relacionaba con la imaginación, lo que pudiera estar asociado a la búsqueda de sensaciones en los niños (Xia et al., 2017). Este estudio no sólo demuestra la activación regional durante el proceso creativo en nińos, sino que lo relaciona con las emociones referidas a tomar riesgos, gratificación, impulsividad y la tendencia a buscar nuevas sensaciones propias de esta edad. Estas observaciones podrían ser útiles para ser empleadas como posibles estrategias para motivar la creatividad en niños.

\section{Genes y creatividad}

También se ha encontrado una relación entre la creatividad y los genes del sistema serotoninérgico, el cual está implicado en la atención, memoria visual, verbal y operativa (Ansburg y Hill, 2003; Peciña et al., 2013; Reuter, Roth, Holve y Hennig, 2006; Wolf, Kulikov, Bortsov y Popova, 2009). En Finlandia se describió un gen candidato para la apreciación y producción musical, vinculado con el del receptor de la vasopresina AVPRIA, que además puede 
estar comprometido con el comportamiento y la cognición social (Ukkola, Onkamo, Raijas y Järvelä, 2009). Estos estudios sugieren una interrelación entre inteligencia y creatividad, que van de la mano hasta cierto punto, para luego poder tomar caminos independientes. Es evidente el juego entre las diversas áreas cerebrales para promover ambos procesos, además, el avance de la genética permite escudriñar e incentivar el rol que puede jugar esta disciplina en el desarrollo de la creatividad.

Juan Sebastián Bach diseñó un árbol genealógico con sus ancestros y descendientes catalogándolos en diferentes grados de habilidad musical (Lehmann, 2018). Este ejemplo, entre otros, alimenta la discusión sobre la herencia de determinadas características como la destreza musical, por un lado, comparado con la adquisición de esas destrezas a través de un ambiente motivador e instigador. Se trata del dilema sobre «si se nace o se hace». Al respecto los estudios de genética aquí presentados han dado una respuesta sorprendente y novedosa a favor de la primera opción. Por supuesto, dichos hallazgos no cierran el dilema, sino que alientan a explotar y explorar los eventuales alcances novedosos de la herencia, la epigenética y la estimulación ambiental.

\section{El papel de la motivación intrinseca y extrinseca}

La creatividad requiere de motivación, inteligencia y apertura a nuevas experiencias. La motivación intrínseca demuestra el gozo individual e intransferible con el que se realiza una actividad, mientras que la motivación extrínseca es una recompensa externa, como un premio, incentivo, reconocimiento o un salario.

El sistema dopaminérgico mesolímbico, mediaría tanto la búsqueda de experiencias nuevas como la motivación interna al facilitar un comportamiento voluntario, propositivo y ávido de recompensa, en tanto que inhibe otros comportamientos en competencia (Flaherty, 2005; Hamid et al., 2016; Mirolli, Santucci y Baldassarre, 2013). Parece que la motivación intrínseca está más relacionada con la creatividad al perseguir una intención propia; por otra parte, se ha probado que una recompensa externa disminuye la magnitud del interés e ímpetu de una actividad propuesta. La motivación intrínseca para 
el aprendizaje se mantiene estable desde la edad escolar hasta la adolescencia y, al reforzarse con la curiosidad exploratoria, se incentiva aún más la creatividad; mientras que la motivación extrínseca media la eficiencia del trabajo (Lepper, Greene y Nisbett, 1973; Deci, 1971; Prabhu, Sutton y Saucer, 2008; Hennessy, Amabile y Martinage, 1989; Bromberg-Martin y Hikosaka, 2009).

\section{El estado de flow}

El estado de motivación intrínseca de mayor expresión atencional se denomina flow. En dicho estado se deja de lado la percepción del entorno, de sí mismo, el trabajo fluye sin mayor exigencia y no existe durante el mismo una sensación de laxitud. Para lograr un estado de flow se requiere reunir las siguientes características:

1. La actividad tiene un objetivo claro.

2. Existe una retroalimentación inmediata sobre la efectividad de la acción.

3. El pensamiento está totalmente ocupado con la acción.

4. No existe temor al fracaso.

5. Las necesidades personales/fisiológicas pasan a un segundo plano $\mathrm{u}$ «olvido».

6. No hay conciencia del tiempo.

7. La actividad tiene una motivación interna.

8. La exigencia de la actividad concuerda con la capacidad de la persona para realizarla.

En algunos estudios de resonancia magnética funcional se encontraron los siguientes hallazgos durante la experiencia de flow:

1. Incremento de la actividad neuronal en el giro frontal anteroinferior y putamen izquierdos que sugieren respectivamente un mayor control cognitivo y éxito probabilístico en la tarea asignada.

2. Reducción de la actividad neuronal en la corteza prefrontal medial y de la amígdala, vinculados respectivamente con una reducción en el proce- 
samiento de la información autorreferencial y disminución de emociones negativas.

3. Los autores de otro estudio relacionan la actividad del putamen durante el estado de flow con afecto positivo, buena autoestima, satisfacción y bienestar psicológico (Dietrich, 2004; Ulrich, Keller, Hoenig, Waller y Grön, 2014; De Manzano et al., 2013).

Hasta ahora la evidencia señala que el flow permite disfrutar de la creatividad, incrementa la producción creativa, pero al parecer no genera las nuevas ideas propias de la creatividad (MacDonald, Byrne y Carlton, 2006; Cseh, Philipps y Pearson, 2015).

El conocimiento progresivo sobre la motivación extrínseca e intrínseca, incluyendo el flow, resulta interesante en la discusión en las áreas de creatividad y del proceso enseñanza-aprendizaje. En los dos tipos de motivación se llega a un producto final que termina siendo evaluado o recompensado. En la motivación intrínseca se presume una motivación innata y un disfrute del proceso voluntario para llegar al producto final. Estos aspectos pueden verse distorsionados al colocárseles una valoración externa como una nota académica o una gratificación monetaria. Es posible entonces que el artista pinte por ganar dinero y no por la motivación propia del arte, o que el alumno estudie por la nota y no por el goce del proceso de aprender. El cómo evitar que la motivación extrínseca pueda echar a perder el proceso creativo o de aprendizaje al interferir con la motivación intrínseca, es otro campo de posibilidades de exploración.

\section{El hemisferio creativo}

Varios estudios neuropsicológicos han demostrado la preponderancia del hemisferio derecho en la creatividad (Beeman y Bowden, 2000; Katz, 1983, 1986). No obstante, varias observaciones con neuroimágenes funcionales sobre improvisación musical, creatividad verbal y lírica, evidencian una activación e inactivación en red de ambos hemisferios (Limb y Braun, 2008; Liu et al., 2012; De Manzano y Ullén, 2012). Incluso algunos estudios electroence- 
falográficos prueban actividad importante en el hemisferio izquierdo durante la acción creativa en pintura, escritura o música, en regiones frontopolares y occipitales, y en el área de asociación parieto-temporo-occipital derecha. Algunos investigadores consideran que el hemisferio derecho expone durante el proceso de creación múltiples propuestas de ideas y asociaciones de manera burda y amplia, mientras que el izquierdo las organiza, cataloga o desecha acorde con la circunstancia. En ambas actividades se emplean tractos cortico-corticales inter e intrahemisféricos, lo que hace patente la interrelación bihemisférica en la creatividad (Petsche, 1996; Jausovec y Jausovec, 2000; Aghababyan et al., 2007).

En resumen, en consonancia con los resultados observados, no existe un hemisferio creativo, sino la interacción de redes neuronales que comprometen ambos hemisferios en el proceso de creación. El aporte de las neuroimágenes funcionales al respecto es importante al demostrar la distribución de funciones en áreas específicas en el proceso creativo.

\section{Eureka}

Algunos artistas y científicos obtienen sus creaciones de manera súbita, casi inesperada, como en una especie de iluminación o revelación. A diferencia del pensamiento divergente, en el que múltiples opciones se abren a una situación, en la intuición —insight - todo el pensamiento se vuelca de manera integral y sencilla a una sola salida o solución abrupta. Esta forma de creatividad se consolida en el adulto, en tanto que el pensamiento divergente es propio del escolar y adolescente. En la primera se activa la corteza prefrontal izquierda y en la intuición el giro temporal superior derecho. Se considera que el giro temporal superior izquierdo cumple la función de categorizar de manera pulcra y específica las relaciones semánticas de un contexto, mientras que su contraparte derecha contribuye a las posibles asociaciones sobre dicho contexto (Kleibeuker, De Dreu y Crone, 2013; Jung-Beeman et al., 2004; Bowden Jung-Beeman, 2003; Chi y Snyder, 2011). Se puede entonces generar una idea creativa de manera estructurada, analítica, juiciosa y programada como en el pensamiento divergente, pero incluso en ese tipo de ejercicio puede inmiscuir- 
se la lucidez inesperada de la intuición, que ofrece la salida definitiva a una situación. Con frecuencia esta iluminación va acompañada de una sensación emocional de asombro y agradable sorpresa.

\section{La imaginación}

La imaginación, figuración o preconcepción sensorial puede ser una forma de creatividad, cuyas reales dimensiones y alcances deben ser exploradas aún. Un metaanálisis al respecto da a la imaginación como fuente creativa, empleando el pensamiento divergente, un pobre $3 \%$. No obstante es común escuchar en personas del común, así como en artistas, científicos y profesionales, que la imaginación es la fuente inspiradora de su creatividad, lo que no concuerda con el resultado presentado en el metaanálisis.

Es posible que la imaginación deba ser estudiada más con métodos de la intuición que con pruebas de pensamiento divergente. Un estudio de neuroimágenes funcionales sobre la imaginación demuestra la activación de áreas con funciones de atención —lóbulo frontal, núcleos basales—, memoria lóbulos temporales-, preparación motora — corteza premotora- y áreas de asociación —corteza temporo-occipito-parietal一.

La imaginación auditiva suprime parcialmente áreas de procesamiento visual y viceversa. La imaginación visual y auditiva disminuye la actividad de las cortezas primarias de la visión y audición (LeBoutillier y Marks, 2003; Shavell, 2013; Zvyagintsev et al., 2013). Como se ha demostrado, la imaginación involucra la coordinación de activación e inactivación simultánea de varias áreas cerebrales, y se requiere mayor estudio de su impacto en el proceso de creación.

Las fuentes de provocación: caminar, el verde, la relajación, la experiencia multicultural y el sueño

Para el proceso de creación es menester tener inspiración y ésta se puede promover de diferentes maneras, como por ejemplo al caminar, rodearse de verde, relajarse, tener contacto multicultural, gesticular o al dormir. 
Un estudio español en escolares evidencia la correlación entre la creatividad y el estado físico de los estudiantes, al igual que la capacidad aeróbica como predictor de creatividad. El análisis por subgrupos destaca que los estudiantes con mayor creatividad tenían mejor estado físico (Latorre, Garcia, Pantoja y Berrios, 2017). Opezzo y Schwartz (2014) prueban cómo la creatividad se incrementa sustancialmente en cuatro pruebas diferentes tras emplear la marcha como mecanismo provocador del funcionamiento cerebral. Se postula que la actividad física facilita la creatividad al:

1. Disipar la función ejecutiva por la multiplicidad de funciones a desarrollar — caminar, vigilar el entorno y crear-.

2. Promover un buen ánimo — por el ejercicio— que incentive el surgimiento de nuevas ideas.

3. Facilitar la relajación en la supresión de memoria que promueve la aparición de nuevas ideas asociativas.

4. Incrementar la función de memoria en el hipocampo (Opezzo y Schwartz, 2014; Baas, De Dreu y Nijstad, 2008; Steinberg, Sykes, Moss, Le Boutillier y Dewey, 1997; Chrysikou y Thompson-Schill, 2011; Erickson et al., 2011).

El desarrollar actividades al aire libre o con un estímulo visual verde también incentiva el potencial creativo según dos estudios. Los autores refieren que un ambiente rico en estímulos naturales puede facilitar el fenómeno creativo. El ambiente natural debe estar alejado de la interrupción constante por herramientas tecnológicas y debe ser emocionalmente tranquilizador para lograr el objetivo creativo; a su vez, desde un planteamiento cognitivo y psicológico, el color verde da la impresión de permisividad para seguir adelante (Atchley, Strayer y Atchley, 2012; Lichtenfeld, Elliot, Maier y Pekrun, 2012).

Acorde con otro artículo, las experiencias multiculturales pueden promover la creación por intuición: la asociación y generación de ideas no habituales y el afloramiento de conocimiento poco convencional de culturas lejanas pueden ser útiles para la expansión de soluciones innovadoras en otro ambiente (Leung, Maddux, Galinsky y Chiu, 2008). 
En los momentos de relajación, distracción u ocio, en los que no se requiere una atención sostenida y dirigida, se activan las cortezas prefrontal medial, del cíngulo y el precúneo, constituyendo la denominada red neuronal por defecto - Default Mode Network (DMN)_. Esta red está implicada en funciones de autoconciencia, autopercepción, autorreferenciación temporal y espacial, introspección, recuperación de la memoria autobiográfica y de los deseos, sueños y miedos. Su actividad permite la ensońación, "el soñar despierto». Su mayor actividad se relaciona con el cociente intelectual, la apertura a nuevas experiencias y la aparición de ideas libres de perjuicio moral; condiciones propicias para la creatividad. La red se activa durante actividades relajantes como caminar o bañarse, precisamente cuando muchas personas pueden tener súbitamente la solución a un problema (Davey, Pujol y Harrison, 2016; Cavanna y Trimble, 2006; Baird et al., 2012; Hearne, Mattingley y Cocchi, 2016; Baety, 2016). La misma red mantiene la actividad durante la etapa del sueño de movimientos oculares rápidos o sueño Mor. Aquellas personas que logran recordar con más frecuencia sus sueños tienen una apertura a experimentar cosas novedosas y una mayor creatividad (Eichenlaub et al., 2014; Watson, 2003). Este conocimiento puede emplearse en los ámbitos familiares, escolares, laborales y de esparcimiento para incentivar de manera propositiva la creatividad.

Otro estudio en la Universidad de York observa cómo la promoción de gesticulación en niños posibilita el pensamiento creativo. Los gestos favorecen las explicaciones no verbales de determinadas ideas, facilitan el cambio de pensamientos y generan nuevas ideas en esta población (Kirk y Lewis, 2017).

Las formas descritas de provocación de la creatividad están relacionadas con un estilo de vida saludable y con el cuidado del medio ambiente. Es conocido el efecto de la actividad física en aspectos físicos y mentales, dando aquí un aporte adicional al propósito creativo. Algo similar ocurre con las actividades de ocio y el sueño. Ambas circunstancias están relacionadas con la relajación, recuperación física y mental, así como con la fijación de la memoria y el aprendizaje. No sobra reforzar la importancia de los estilos de vida saludable en una sociedad y en algunos sistemas educativos, los cuales han disminuido progresivamente las horas semanales de actividad física, sueño, 
ocio y el tiempo dedicado a las artes y las ciencias sociales. Las actividades de esparcimiento al aire libre y en la naturaleza también se han reducido o se ven amenazadas por varias razones. A su vez, se observa una mayor dedicación de todos los ciudadanos, en especial de niños y adolescentes, a esparcimientos sedentarios en ambientes cerrados como en el caso de la exposición creciente a pantallas (Pan American Health Organization, 2018; Guerra y Rodríguez, 2018; Silió y Torres, 2018; Hillman, Kamijo y Scudder, 2011; García y Piqueras, 2019). Las observaciones realizadas en los estudios arriba mencionados tienen la particularidad de ser a corto plazo. El efecto sobre la creatividad con una intervención continua queda aún por determinar en lapsos prolongados.

\section{Conclusiones}

El trabajo de producir algo novedoso y útil en un contexto social se denomina creatividad y es, junto con la inteligencia social, un rasgo distintivo del ser humano respecto a otras especies animales. Como actividad mental la creatividad puede ser estudiada por la ciencia cognitiva con múltiples pruebas, que aunadas al avance tecnológico de las neuroimágenes funcionales y la genética pueden dar luces para la comprensión e intervención propositiva de la creatividad. Se demostró la interrelación entre creatividad e inteligencia, motivación, imaginación y la apertura a nuevas experiencias. Las neuroimágenes funcionales quiebran el paradigma de un hemisferio creativo $y$, antes bien, evidencian la coordinación funcional en esta actividad. La genética ha aportado indicios de que algunos rasgos creativos pueden ser heredados. Se destacan los mecanismos provocadores de creatividad como herramientas disponibles en todo lugar, tales como la actividad física, sueño, ocio, exposición al verde o a la naturaleza o la interculturalidad. Quedan abiertas varias opciones de investigación como por ejemplo la elaboración de pruebas de medición de creatividad acorde con el avance de las ciencias, las artes y las tecnologías y su interrelación con los diferentes tipos de inteligencia o los estados emocionales, así como el efecto a largo plazo sobre la creatividad de intervenciones facilitadoras. 
Los hallazgos encontrados sobre la génesis y fomento de la creatividad pueden tener un impacto importante en todas las profesiones y oficios del ser humano, y especialmente en las ciencias humanas, las artes y la educación. No se deben excluir las áreas de las ciencias exactas y naturales. El desafío, no sólo radica en ahondar en el conocimiento sobre la creatividad, sino precisamente en la traslación de esos conocimientos en la práctica de nuestro quehacer cotidiano.

\section{REFERENCIAS}

Aghababyan, A., Grigoryan, V., Stepanyan, A., Arutyunyan, N., y Stepanyan, L. (2007). Eeg Reactions During Creative Activity. Human Physiology, 33, 252253.

Ansburg, P., y Hill, K. (2003). Creative and Analytic Thinkers Differ in their Use of Attentional Resources. Personality and Individual Differences, 34, 1141-1152.

Atchley, R., Strayer, D., y Atchley, P. (2012). Creativity in the Wild: Improving Creative Reasoning through Immersion in Natural Settings. PLoS ONE, 7(12), e 51474 .

Baas, M., De Dreu, C., y Nijstad, B. (2008). A Meta-analysis of 25 years of Mood - Creativity Research: Hedonic Tone, Activation, or Regulatory Focus? Psychological Bulletin, 134, 779-806.

Baety, R., Kaufman, S., Benedek, M., Jung, R., Kenett, Y., Jauk, E., Neubauer A., y Silvia, P. (2016). Personality and Complex Brain Networks: the Role of Openness to Experience in Default Network Efficiency. Human Brain Mapping, 37(2), 773-779.

Baird, B., Smallwood, J., Mrazek, M., Kam, J., Franklin, M., y Schooler, J. (2012). Inspired by Distraction: Mind Wandering Facilitates Creative Incubation. Psychological Science, 23(10) 1117-1122.

Beeman, M., y Bowden, E. (2000). The right hemisphere maintains solution-related activation for yet-to-be-solved problems. Memory \& Cognition, 28(7), 1231-1241.

Bowden, E., y Jung-Beeman, M. (2003). Aha! Insight Experience Correlates with Solution Activation in the Right Hemisphere. Psychonomic Bulletin Review, 10, $730-737$. 
Bromberg-Martin, E., y Hikosaka, O. (2009). Midbrain Dopamine Neurons Signal Preference for Advance Information about Upcoming Rewards. Neuron, 63(1), 119-126.

Carlsson, I., Wendt, P., y Risberg, J. (2000). On the Neurobiology of Creativity. Differences in Frontal Activity between High and Low Creative Subjects. Neuropsychologia, 38, 873-885.

Cavanna, A., y Trimble M. (2006). The Precuneus: a Review of its Functional Anatomy and Behavioural Correlates. Brain, 129, 564-583.

Chi, R., y Snyder, A. (2011). Facilitate Insight non-invasive Brain Stimulation. PLoS ONE, 6(2), e16655.

Chrysikou, E., y Thompson-Schill, S. (2011). Dissociable Brain States linked to Common and Creative Object Use. Human Brain Mapping, 32, 665-675.

Cseh, G., Phillips, L., y Pearson, D. (2015). Flow, Affect and Visual Creativity. Cognition \& Emotion, 29, 281-291.

Davey, C., Pujol, J., y Harrison, B. (2016). Mapping the Self in the Brain's default Mode Network. NeuroImage, 132, 390-397.

Deci, E. (1971). Effects of Externally Mediated Rewards on Intrinsic Motivation. Journal of Personality and Social Psychology, 18, 105-115.

De Manzano, Z., y Ullén, Ö. (2012). Goal-independent Mechanisms for Free Response Generation: Creative and Pseudo-random Performance Share Neural Substrates. NeuroImage, 59, 772-780.

De Manzano, O., Cervenka, S., Jucaite, A., Hellenäs, O., Farde, L., y Ullén, F. (2013). Individual Differences in the Proneness to Have Flow Experiences are Linked to Dopamine D2-receptor Availability in the Dorsal Striatum. NeuroImage, 67, 1-6.

DeYoung, C., Peterson, J., y Higgins, D. (2005). Sources of Openness/Intellect: Cognitive and Neuropsychological Correlates of the fifth Factor of Personality. Journal of Personality, 73, 825-858.

Dietrich, A. (2004). Neurocognitive Mechanisms Underlying the Experience of Flow. Consciousness and Cognition, 13, 746-761.

Dietrich, A. (2004). The Cognitive Neuroscience of Creativity. Psychonomic Bulletin Review, 11(6), 1011-1026.

Eichenlaub, J., Nicolas, A., Daltrozzo, J., Redouté, J., Costes, N., y Ruby P. (2014). Resting Brain Activity Varies with Dream Recall Frequency between Subjects. Neuropsychopharmacology, 39, 1594-1602. 
Erickson, K., Voss, M., Prakash, R., Basak, C., Szabo, A., Chaddock, L., y Kramer, A. (2011). Exercise Training Increases the Size Hippocampus and Improves Memory. Proceedings of the National Academy of Sciences of the United States, 108, 3017-3022.

Flaherty, A. (2005). Frontotemporal and Dopaminergic control of idea Generation and Creative Drive. Journal of Comparative Neurology, 493, 147-153.

Geake, J., y Hansen, P. (2005). Neural Correlates of Intelligence as Revealed by fMrI of Fluid Analogies. NeuroImage, 26(2), 555-564.

García R., y Piqueras M. (11 de febrero de 2019). El arte y su pedagogía. El País. Recuperado de http//www.elpais.com/

Guerra J., y Rodríguez E. (13 de julio de 2018). Una asignatura pendiente: la filosofía en la secundaria. El País. Recuperado de http//www.elpais.com/

Hamid, A., Pettibone, J., Mabrouk, M., Hetrick, V., Schmidt, R., Van der Weele, C., Kennedy, R., Aragona, B., y Berke, D. (2016). Mesolimbic Dopamine Signals the Value of Work. Nature Neuroscience, 19, 117-126.

Hearne, L., Mattingley, J., y Cocchi, L. (2016). Functional Brain Networks Related to Individual Differences in Human Intelligence at rest. Scientific Reports, 6, 323-328.

Hennessy, B., Amabile, T., y Martinage, M. (1989). Immunizing Children against the Negative Effects of Reward. Contemporary Educational Psychology, 14, 212-227.

Hillman C., Kamijo K., y Scudder M. (2011). A Review of Chronic and Acute Physical Activity Participation on Neuroelectric Measures of Brain Health and Cognition During Childhood. Preventive Medicine, 52, 21-28.

Jausovec, N., y Jausovec, K. (2000). EEg Activity during the Performance of Complex Mental Problems. International Journal Psychophysiology, 36, 73-88.

Jung-Beeman, M., Bowden, E., Heberman, J., Frymiare, J., Arambel-Liu, S., Greenblatt, R., Reber, B., y Kounios, J. (2004). Neural Activity when People Solve Verbal Problems with onsight. Public Library of Science Biology, 2(4), 500-510.

Jung, R., Segall, J., Bockholt, H., Flores, R., Smith, S., Chavez, R., y Haier, R. (2010). Neuroanatomy of Creativity. Human Brain Mapping, 31, 398-409.

Jung, R., Gasparovic, C., Chavez, R., Flores, R., Smith, S., Caprihan, C., y Yeo, R. (2009). Biochemical Support for the "Threshold" Theory of Creativity: A Magnetic Resonance Spectroscopy Study. The Journal of Neuroscience, 29(16), 5319-5325. 
Katz, A. (1983). Creativity and Individual Differences in Asymmetric Cerebral Hemispheric Functioning. Empirical Studies of the Arts, 1, 3-16.

Katz, A. (1986). The Relationships Between Creativity and Cerebral Hemisphericity for Creative Architects, Scientists, and Mathematicians. Empirical Studies of the Arts, 4, 97-108.

Kirk, E., y Lewis Carine. Gesture Facilitates Children's Creative Thinking. Psychological Science, 28(2), 225-232.

Kleibeuker, S., De Dreu, C.., y Crone, E. (2013). The development of Creative Cognition across Adolescence: Distinct Trajectories for insight and Divergent Thinking. Developmental Science, 16(1), 2-12.

Latorre, P., Garcia, F., Pantoja, A., y Berrios, B. (2017). Creativity and physical fitness in primary school-aged children. Pediatrics International, 59, 1194-1199.

LeBoutillier, N., y Marks, D. (2003). Mental Imagery and Creativity: A Meta-Analytic Review Study. British Journal of Psychology, 94, 29-44.

Lehmann, K. (2018). Der erste Tag: Was ist Kreativität? En K, Lehmann (ed), Das schöpferische Gehirn Auf der Suche nach Kreativität - eine Fahndung in sieben Tagen, 1-30. Berlin: Springer-Verlag.

Lepper, M., Greene, D., y Nisbett, R. (1973). Undermining Children's Intrinsic Interest with Extrinsic Reward: a Test of the "Overjustification" Hypothesis. Journal of Personality and Social Psychology, 28, 129-137.

Leung, A., Maddux, W., Galinsky, A., y Chiu, C. Multicultural Experience Enhances Creativity: the When and How. American Psychology, 63, 169-181.

Lichtenfeld, S., Elliot, A., Maier, M., y Pekrun, R. (2012). Fertile Green: Green Facilitates Creative Performance. Psychological Bulletin, 38(6), 784-797.

Limb CJ, Braun AR (2008) Neural Substrates of Spontaneous Musical Performance: An fMri Study of Jazz Improvisation. PLoS One, 3(2): e1679.

LLiu, S., Chow, H., Xu, Y., Erkkinen, M., Swett, K., Eagle, M., Rizik-Baer, D., y Braun, A. (2012) Neural Correlates of Lyrical Improvisation: a fMrI Study of Freestyle Rap. Scientific Reports, 2, 1-8.

MacDonald, R., Byrne, C., y Carlton, L. (2006). Creativity and Flow in Musical Composition: an Empirical Investigation. Psychology of Music, 34, 292-306.

Miller, I, y Tal, I. (2007). Schizotypy versus Openness and Intelligence as Predictors of Creativity. Schizophrenia Research, 93, 317-324. 
Mirolli, M., Santucci, V., y Baldassarre, G. (2013). Phasic Dopamine as a Prediction Error of Intrinsic and Extrinsic Reinforcements Driving both Action Acquisition and Reward Maximization: A Simulated Robotic Study. Neural Networks, 39, 40-51.

Mula, M., Hermann, B., y Trimble, M. (2016). Neuropsychiatry of Creativity. Epilepsy \& Behaviour, 57, 225-229.

Opezzo, M., y Schwartz, D.. (2014). Give your Ideas some Legs: the Positive Effect of Walking on Creative Thinking. Journal of Experimental Psychology, 40(4), 1142-1152.

Pan American Health Organization (2018). The Health of Adolescent and Youth in the Americas. Implementation of the Regional Strategy and Plan of Action on Adolescent and Youth Health 2010-2018. Washington: Pan American Health Organization.

Peciña, M., Mickey, B., Love, T., Wang, H., Langenecker, S., Hodgkinson, C., y Zubieta, J. (2013). DRD2 Polymorphisms Modulate Reward and Emotion Processing, Dopamine Neurotransmission and Openness to Experience. Cortex, 49(3), 877-890.

Petsche, H. (1996). Approaches to Verbal, Visual and Musical Creativity by EEG Coherence Analysis. International Journal of Psychophysiology, 24, 145-159.

Prabhu, V., Sutton, C., y Saucer, W. (2008). Creativity and Certain Personality Traits: Understanding the Mediating Effect of Intrinsic Motivation. Creativity Research Journal, 20, 53-66.

Reuter, M., Roth, S., Holve, K., y Hennig, J. (2006). Identification of First Candidate Genes for Creativity: A Pilot Study. Brain Research, 1069, 190-197.

Shavell, H. (2013). Mental imagery: The key to Dental Artistry. Journal of Esthetic Restoration, 25(1), 4-15.

Silió, E., y Torres, A. (18 de octubre de 2018). El Congreso pacta que la Filosofía vuelva a ser obligatoria tres años en los institutos. El País. Recuperado de https:// elpais.com/sociedad/2018/10/17/actualidad/1539790211_552468.html

Steinberg, H., Sykes, E., Moss, T., Le Boutillier, N., y Dewey, A. (1997). Exercise Enhances Creativity Independently of Mood. British Journal of Sports Medicine, 31, 240-245.

Takeuchi, H., Taki, Y., Hashizume, H., Sassa, Y., Nagase, T., Nouchi, R., y Kawashima, R. (2011). Cerebral Blood Flow during Rest Associates with General Intelligence and Creativity. PLoS One, 6(9): e25532. 
Ukkola, L., Onkamo, P., Raijas, P., y Järvelä, L. (2009). Musical Aptitude is Associated with AVPR1A-Haplotypes. PLoS One 4(5): e5534.

Ulrich, M., Keller, J., Hoenig, K., Waller, C., y Grön, G. (2014). Neural Correlates of Experimentally Induced Flow Experiences. NeuroImage, 86, 194-202.

Watson, D. (2003). To Dream, Perchance to Remember: Individual Differences in Dream Recall. Personality and Individual Differences, 34, 1271-1286.

Wolf, N., Kulikov, A., Bortsov, C., y Popova, N. (2009). Association of Verbal and Figural Creative Achievement with Polymorphism in the Human Serotonin Transporter Gene. Neuroscience Letters, 463, 154-157.

Xia, Y., Zhuang, K., Sun, J., Chen, Q., Wei, D., Yang, W., y Qiu, J. (2017). Emotion-related Brain Structures Associated with Trait Creativity in Middle Children. Neuroscience Letters, 658, 182- 188.

Zvyagintsev, M., Clemens, B., Chechko, N., Mathiak, K., Sack, A., y Mathiak, K. Brain Networks Underlying Mental Imagery of Auditory and Visual Information. European Journal of Neuroscience, 37, 1421-1434. 